\title{
Shielding Analysis and Design for the Undersea Contactless Power Transmission System
}

\author{
Hairong Wang ${ }^{1, \text { a }}$, Zesong $\mathrm{Li}^{2, \mathrm{~b}, \text { * }}$ \\ ${ }^{1}$ Zhoushan Institute of Calibration and Testing for Quality and Technology Supervision, Zhoushan \\ 316021, China \\ ${ }^{2}$ Ningbo Institute of Measuring and Testing, Ningbo 315048, China \\ awhrio@126.com, besonlee@gmail.com
}

Keywords: Contactless power, transmission.

\begin{abstract}
A contactless power transmission system work based on high-frequency electromagnetic induction, companying amount of leakage magnetic flux spread outside the coupling structure and inducing power loss in the metal shell. It is necessary to design shielding mechanism to weaken the influence of leakage magnetic flux. This paper analyzed the shielding effectiveness of metal layer in the time-varying change magnetic field, and revealed the shielding function of a thin copper sheet between cores and shells. By using FEM simulation, distributions of magnetic intensity and eddy current density were imaged and compared to demonstrate the shielding effectiveness. Finally, a prototype of coupling structure with shielding sheets was proposed and tested. The testing results proved the significant affection of shielding sheet on improving the system's transferring ability.
\end{abstract}

\section{Introduction}

The contactless power transmission (CPT) system is suitable for undersea application because of its special structure and advantages, including simple docking, without wearing, little operating force, being immune from electric shock and leakage, and so on ${ }^{[1,2]}$. But the undersea environment is harsh to a CPT system especially in deep sea ${ }^{[3,4]}$. The power interface mainly comprises of ferrite cores and windings, which are fragile in this circumstance being faced with unpredictable impacts. In order to protect working components from damage, metal shells usually made of stainless steel have to be used to make the structure strong enough. The shell is maybe nonmagnetic, but is electric conductive. Generally, a CPT system works at a high frequency and produces quit a few magnetic fluxes in vicinity. Thence, high eddy current will occur in the thick and conductive shell, causing power loss. An actual method to reduce this kind of power loss is setting shielding layer between cores and shells.

Electromagnetic shielding is an inevitable matter in high frequency electric equipment's design, for the high frequency current in circuits always produces a great deal radiation. To meet the electromagnetic compatibility (EMC) requirements, electromagnetic shielding skill is necessary to reduce power loss and electromagnetic interference (EMI) ${ }^{[5]}$. For a CPT system used in undersea environment, the shielding subject is leakage flux caused by the gap between cores.

In this paper, magnetic field in cores and vicinity was analyzed, especially that near the gap and outside cores. By using shielding sheets between cores and shells, the field changes were revealed by imaging flux and intensity vector distribution, and the shielding effectiveness (SE) was evidenced. After that, a more profound study on power loss in shells and shielding layers was practiced to quantify the shielding effectiveness of this method according to the current frequency. Eddy currents in shielding sheet and shells were both simulated by FEM software, and power loss was also calculated to indicate the shielding function. A prototype was design based on the above analysis, and was tested. The testing result was in agreement with the analysis.

\section{Shielding Effective of the Metal layer}

Nonmagnetic metals such as copper, stainless steel cannot be affected by static magnetic field, and the magnetic flux can travel through the entities without any impediment. But in a changing magnetic 
field, free electrons in metals will be forced to move by induced electric field. According to Faraday's law, a time-varying magnetic field produces an electromotive force, and a current may be established in a suitable closed circuit ${ }^{[6]}$. In a CPT system, leakage flux pass through the shells near the gap, and form an equivalent 'closed circuit' for the induced current which is usually called eddy current. On the other hand, the eddy current produces a magnetic field which is opposite to the primary magnetic field, according to Biot-Savart law ${ }^{[6]}$. Hence, the total magnetic field is the composition of the two fields, and the magnetic intensity near shells is weakened. This is the basic electromagnetic shielding principle by using metal layer.

However the purpose of shells is not shielding, but improving structure's strength. The thick wall of shells will result in critical eddy current and power loss. So another conductive layer should be set between cores and shells to weaken the magnetic affection on shells. Fig. 1 show the comparison of magnetic field of a coupling structure with shells only and the other one with shielding sheets between cores and shells. It is obvious that the shielding sheets have efficiently constricted magnetic flux to cores. It is beneficial to the system to improve transferring capability.

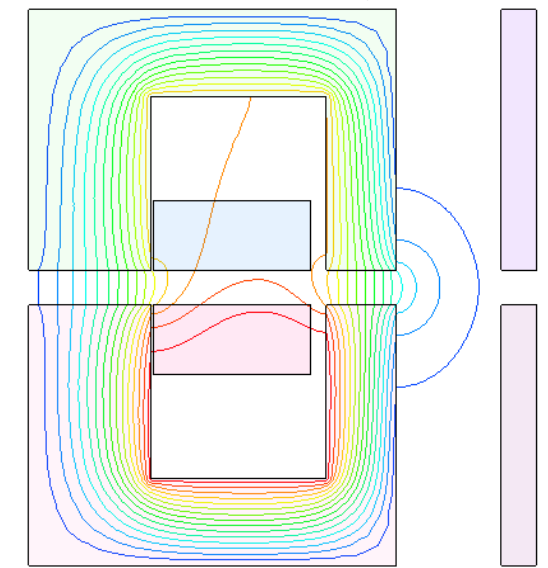

(a)

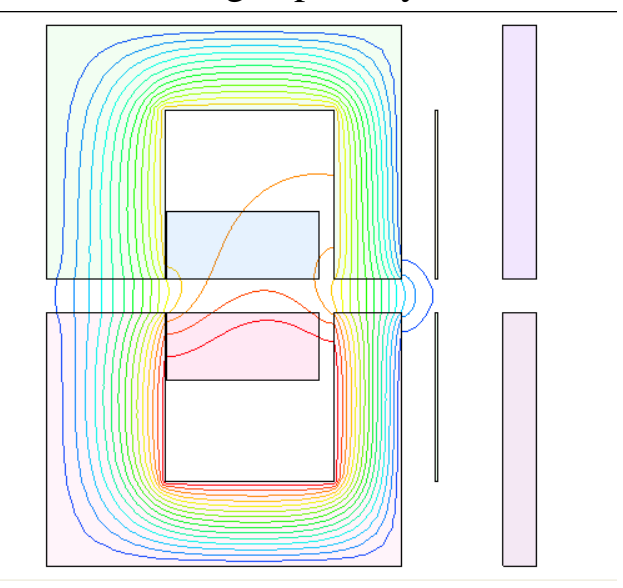

(b)

Fig. 1 The shielding effect on magnetic flux

According to Mexwell theory, a time-varying magmetic field produce a time-varying electric field, and vice versa. So the two kinds of fields have a relationship as following,

$$
\begin{aligned}
& \nabla \times \vec{E}=-\frac{\partial \vec{B}}{\partial t} \\
& \nabla \times \vec{H}=\frac{\partial \vec{D}}{\partial t}+\vec{J}
\end{aligned}
$$

Where, $\vec{E}$ is the electric intensity vector, $\vec{B}$ is the magnetic flux density vector, $\vec{H}$ is the magnetic intendity vector $\vec{D}$ is the electric displacement vector, $\vec{J}$ is the current density vector, $\nabla$ is the Hamilton operator. If the electromagnetic field is sinusoidal with angle frequency $\omega$, the above equations can be rewriten based on Euler transform,

$$
\begin{aligned}
& \nabla \times \vec{E}=-j \omega \vec{B} \\
& \nabla \times \vec{H}=j \omega \vec{D}+\vec{J}
\end{aligned}
$$

Where, $\mathrm{j}$ is the imaginary unit.

Taking the constitutive relationship of $\vec{E}$ and $\vec{J}, \vec{B}$ and $\vec{H}$, the following equation is deduced from (3) and (4),

$$
\vec{H}=-\frac{1}{j \omega \mu \sigma} \nabla \times \vec{J}
$$

Where, $\mu$ is magnetic permeability, $\sigma$ is the conductivity. This equation illustrates how the eddy current affect magnetic field. According to equations (1) and (3), the eddy current is perpendicular to magnetic flux density vector, and proportional to the time rate of change of flux. According to 
equation (5), in conductive parts, such as shells and shielding sheets, magnetic intendity $\vec{H}$ is hold back by eddy current, and is reversly proportional to frequency and conductivity. As a result, the outer side of the structure from shells or shielding sheets is little affected by the primary magnetic flux. Fig.2 shows the distribution of magnetic intendity vectors in the structure, in which (a) is with shells only, (b) with shells and shielding sheets. The sheets are made of copper and their thickness is no larger than the depth of penetration based on the current frequency ${ }^{[7]}$. This figure demonstrates shielding effectiveness of shielding sheets, which prevent most leakage flux from interference to the outer side of the structure, and the result is corresponding to the above analysis.

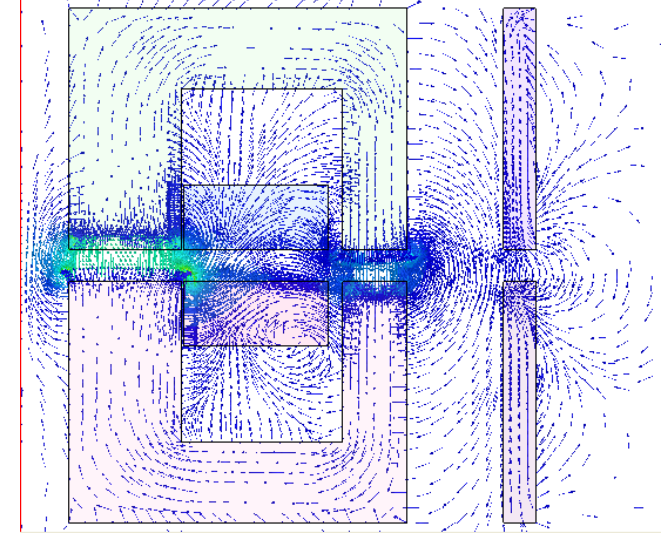

(a)

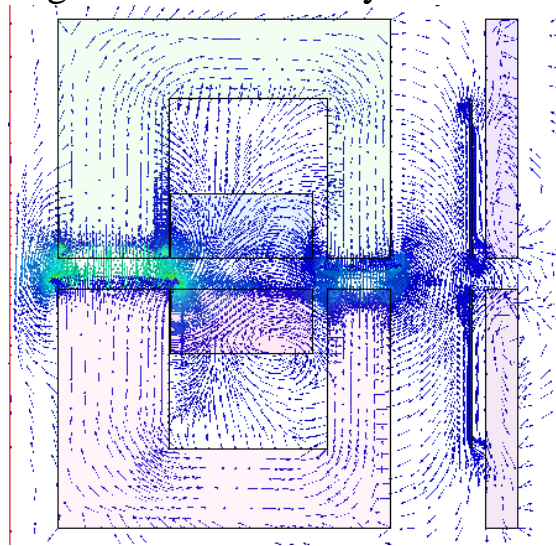

(b)

Fig.2 Magnetic field intensity vectors distrubution of the coupling structure

\section{Analysis of Power Loss in Shells and Shielding Sheets}

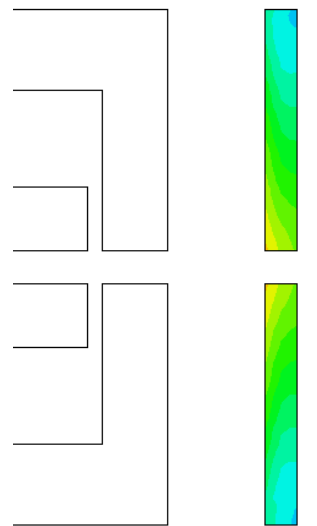

(a)

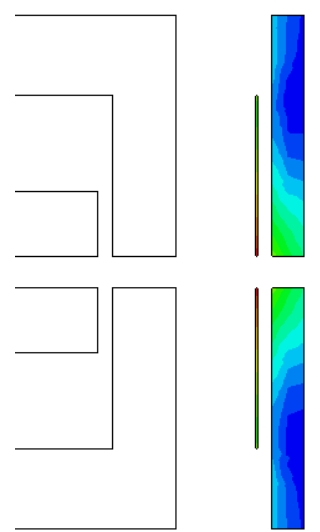

(b)

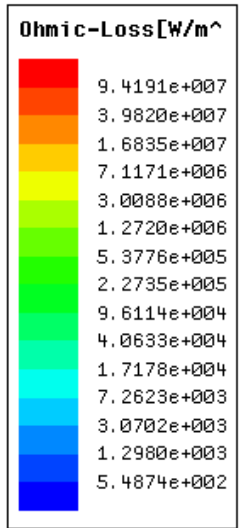

Fig.3 Power loss in shielding (a)without shielding sheet; (b) with thin copper shielding sheet

Another aspect should be considered in electromagnetic shielding is power loss in metal parts, because eddy current in shells and sheets is inevitable. As aforementioned, the shielding sheet can effectively reduce EMI in the outer side of the strucure. But eddy current in it also causes power loss. By using FEM software Ansoft Maxwell, Fig.3 make the eddy currents in shells and shielding sheets visible. The eddy current density in shells shown in Fig.3 (b) is much smaller than that shown in Fig.3 (a), due to copper sheets in front of shells. The eddy current in the sheets is also displayed in the figure, and the dendity is higher than that in shells because the sheets are closer to cores and exposed in more intense leakage flux. Nevertheless, the sheets are very thin, only $0.05 \mathrm{~mm}$, thus the eddy current is not so large. Therefore, power loss in shielding sheets is much smaller than that it saved in shells. This effect can be seen in Fig.4 which calculated power loss caused by eddy currents in Fig.3., where $N_{P}$ and $N_{S}$ are the primary and secondary windings turns respectively, and $I_{P}$ is the primary current. The power-loss curves both increase from low frequency region to high frequency region, but the shielding one is always below the other one. This result demonstrate that the shielding sheets in this structure is significant, especially when the current frequency is very high. 


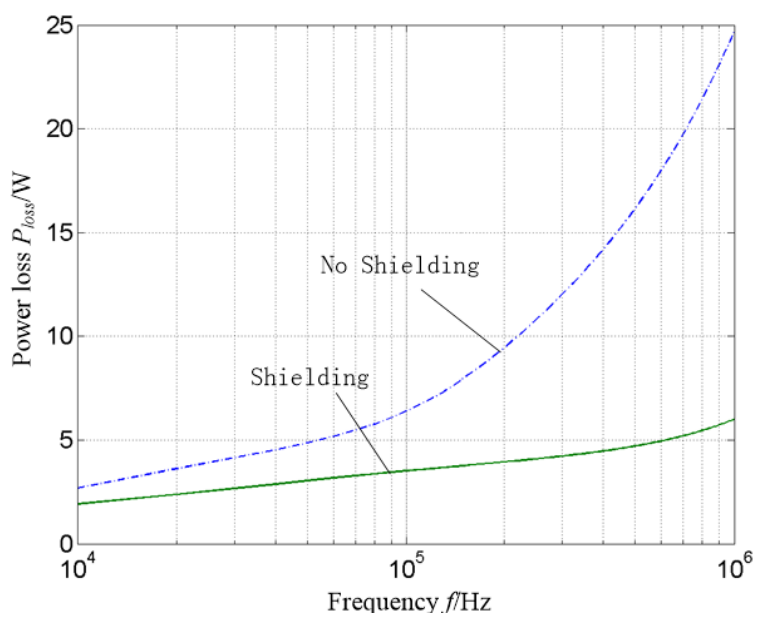

Fig.4 Power loss in shielding with different frequencies(NP=NS=30,IP=5A)

\section{Prototype and Testing}

As suggested above, a prototype of the coupling structure was fabricated with a $0.05 \mathrm{~mm}$ copper sheet outside the ferrite core. One part of this prototype is shown in Fig.5. Additonally, a stainless-steel shell was also prepared for it. Finally, some experiments were performed to test the shielding effectvieness. Input power and output power were both recorded to calculate transferring efficiencies. The results are shown in Fig. 6 which includes four curves representing four different transferring states specialized in the figure. It can been seen that the shelled strucuture has most power loss and lowest efficiency and the shielding sheets can improve its efficiency to a large degree.

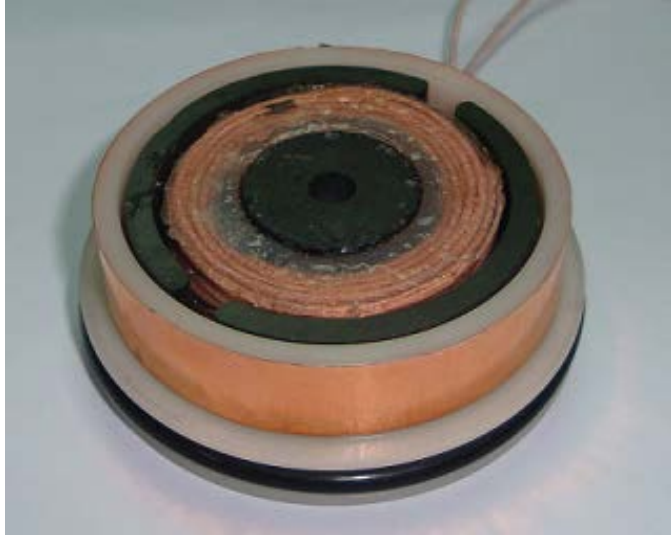

Fig.5 The EM shielding configuration of the coupler

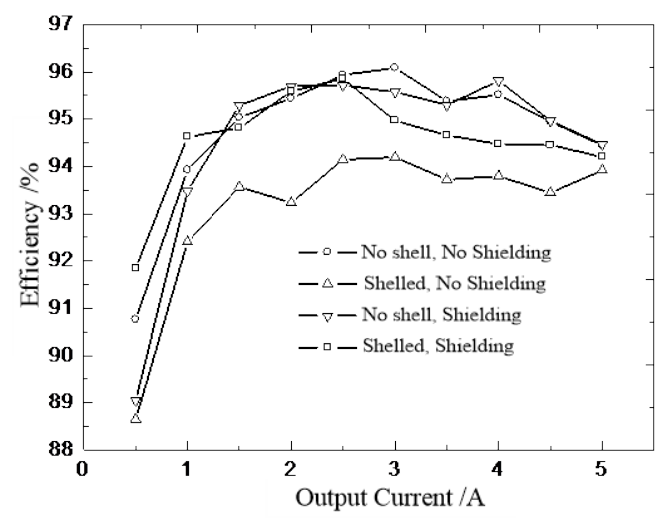

Fig.6 Transferring efficiencies in experiments

\section{Summary}

In this paper, we investigated the electromagnetic shielding effectiveness in a coupling structure with metal shells and shielding sheets. The eddy current was analyzed based on electromagnetic induction theory, and its effect on the primary magnetic field was also revealed. By using FEM simulation, the distribution of magnetic intensity was visualized to prove the shielding effectiveness. Power loss and eddy current density in shells and shielding sheets were also compared to demonstrate the significant function of shielding sheets. As a result, a prototype of coupling structure with shielding sheets was fabricated and tested. The testing data proved that the designed shielding mechanism can improve the transferring efficiency effectively.

\section{Acknowledgements}

This work was supported by National Science foundation of Zhejiang Province (LY12E05004) and Shaoxing Public Technology Appling Plan (2013B70008). Furthermore, we would like to express 
our gratitude to professor Li Dejun in Zhejiang University, for his instructive advice and providing research hardware and software for this paper.

\section{References}

[1] T. Kojiya, F. Sato, H. Matsuki et al., Construction of non-contacting power feeding system to underwater vehicle utilizing electro magnetic induction. in Oceans 2005 - Europe, 2005, Vols 1 and 2, pp. 709-712.

[2] Z.S. Li, D.J. Li, L. Lin, Y. Chen, Design Considerations for EM couplers in CLPT systems for Deep-sea Applications. Journal of Zhejiang University- Science C, 2010,11(10):824-834.

[3] T. Kojiya, F. Sato, H. Matsuki et al., Construction of non-contacting power feeding system to underwater vehicle utilizing electro magnetic induction. in Oceans 2005 - Europe, 2005, Vols 1 and 2, pp. 709-712.

[4] Z.S. Li, D.J. Li, L. Lin, Y. Chen, Design Considerations for EM couplers in CLPT systems for Deep-sea Applications. Journal of Zhejiang University- Science C, 2010,11(10):824-834.

[5] S.C. Tang, S.Y. Hu, H.S.H. Chung, Evaluation of the shielding effects on printed circuit-board transformers using ferrite plates and copper sheets. IEEE Transactions on Power Electronics, 2002,17(6):1080-1088.

[6] W.H. Hayt, J. A. Buck, Engineering Electromagnetic, sixth ed., McGraw-Hill, New York,2002. W. Strunk Jr., E.B. White, The Elements of Style, third ed., Macmillan, New York, 1979.

[7] K.D.Papastergiou, A power converter with a rotating secondary stage for an airborne radar system. Phd Thesis, Edinburgh: University of Edinburgh, 2005. 